PROCEEDINGS OF THE

AMERICAN MATHEMATICAL SOCIETY

Volume 127, Number 9, Pages 2633-2642

S 0002-9939(99)05406-

Article electronically published on May 19, 1999

\title{
EXISTENCE OF TWISTOR SPACES OF ALGEBRAIC DIMENSION TWO OVER THE CONNECTED SUM OF FOUR COMPLEX PROJECTIVE PLANES
}

\author{
F. CAMPANA AND B. KREUSSLER \\ (Communicated by Ron Donagi)
}

\begin{abstract}
We prove the existence of twistor spaces of algebraic dimension two over the connected sum of four complex projective planes $4 \mathbb{C P}^{2}$. These are the first examples of twistor spaces of algebraic dimension two over a simply connected Riemannian four-manifold with positive scalar curvature. For this purpose we develop a method to distinguish between twistor spaces of algebraic dimension one and two by looking at the order of a certain point in the Picard group of an elliptic curve.
\end{abstract}

\section{INTRODUCTION}

Twistor spaces were introduced by R. Penrose [Pe]. The first rigorous mathematical foundation of these ideas was given by M. Atiyah, N. Hitchin and I. Singer in their now classical paper [AHS]. The twistor construction gives a close relationship between three-dimensional complex geometry and real four-dimensional conformal self-dual geometry.

From our point of view, a twistor space $Z$ is a complex three-manifold with the following additional structure:

- A proper differentiable submersion $\pi: Z \rightarrow M$ onto a real differentiable fourmanifold $M$. The fibres of $\pi$ are holomorphic curves in $Z$ being isomorphic to $\mathbb{C P}^{1}$ and having normal bundle in $Z$ isomorphic to $\mathcal{O}(1) \oplus \mathcal{O}(1)$.

- An anti-holomorphic fixed-point free involution $\sigma: Z \rightarrow Z$ with $\pi \sigma=\pi$.

Note that $\pi$ is not holomorphic ( $M$ carries, in general, no almost complex structure). The fibres of $\pi$ are called "real twistor lines" and the involution $\sigma$ is called the "real structure". Anything which is invariant under $\sigma$ will obtain the attribute "real". For example, a complex subvariety $D \subset Z$ is "real" if $\sigma(D)=D$. We shall only consider twistor spaces which are both compact and connected.

The first classification results are due to N. Hitchin [H2] and T. Friedrich, H. Kurke $[\mathrm{FK}]$. They showed that there exist exactly two compact twistor spaces which are Kählerian. Such a twistor space $Z$ is automatically Fano (that is: $-K_{Z}$ is ample, so that $Z$ is, in particular, projective). The corresponding Riemannian

Received by the editors June 20, 1996.

1991 Mathematics Subject Classification. Primary 32L25, 32J17, 32J20.

(C)1999 American Mathematical Society 
four-manifolds are the ${ }^{4}$-sphere $S^{4}$ and the complex projective plane $\mathbb{C P}^{2}$ (with Fubini-Study metric).

New examples of twistor spaces of algebraic dimension three (that is, Moishezon) were first produced by Y.S. Poon [Po1]. There the manifold $M$ was $2 \mathbb{C P}^{2}$. The corresponding $Z$ are not projective or even Kähler, although they are bimeromorphic to $\mathbb{P}^{3}$.

A partial generalization of the results of [H2] and [FK] was obtained in [C2], where it is shown that if $Z$ is a twistor space of the class $\mathcal{C}$ (that is, bimeromorphic to some compact Kähler manifold), then $Z$ is Moishezon and simply connected. This implies, using deep results of M. Freedman [F] and S. Donaldson [Don], that $M$ is homeomorphic to $n \mathbb{C P}^{2}$ for some $n \geq 0$.

In complete anology with the Kähler case, it was also established in [Po2] that if $Z$ is a simply connected twistor space, its algebraic dimension is equal to $\kappa\left(Z, K_{Z}^{-1}\right)$, the Iitaka dimension of its anti-canonical bundle. Hence, if $Z$ is Moishezon, it is weakly Fano (that means $\kappa\left(Z, K_{Z}^{-1}\right)=3$ ). From results of M. Ville [V] and P. Gauduchon [G] it follows that "simply connected" can be relaxed to $b_{1}(Z)=0$ in the result of [Po2].

The existence of twistor spaces $Z$ over $M=n \mathbb{C P}^{2}$ was shown in [DonF] for $n=4$ with $a(Z)=1$ and for $n \geq 5$ with $a(Z)=0$. The first explicit examples of twistor spaces for $n \geq 3$ were constructed by C. LeBrun [LeB1]. We follow the literature and call these spaces LeBrun twistor spaces. They have algebraic dimension three and their complex geometry was studied by H. Kurke $[\mathrm{Ku}]$. Recently, Pedersen and Poon have shown [PP3] that (for fixed $n$ ) LeBrun's and Donaldson-Freedman's examples belong to the same deformation family.

The first example of a twistor space of algebraic dimension two was found by M. Pontecorvo [Pon1]. These twistor spaces are of positive type but not simply connected. The corresponding self-dual Riemannian four-manifold is a primary Hopf surface (equipped with negative orientation) which has odd first Betti number. We present here the first example of a simply connected twistor space having algebraic dimension two.

It seems to be natural to consider the following

Problems. (i) What are the possible algebraic dimensions of twistor spaces $Z$ over $M=n \mathbb{C P}^{2}$ ?

(ii) Compute the algebraic dimension of such twistor spaces $Z$ in terms of divisors on $Z$.

There are several results concerning these problems. To explain them, we need the notion of the "type" of a twistor space. To define this notion, we look at the self-dual conformal structure induced on $M$ by the twistor space. By a result of R. Schoen [Sch], every conformal class of a compact Riemannian four-manifold contains a metric of constant scalar curvature. Its sign will be called the type of the twistor space. This is an invariant of the conformal class, hence of the twistor space.

In this paper we focus on the case of positive type. This is because in this case the vanishing theorem of Hitchin (2.1) can be applied. Furthermore, note that a twistor space of algebraic dimension two is of positive type [Pon2].

On the other hand, Poon's computation of the algebraic dimension [Po3] together with the Riemann-Roch formula and Hitchin's vanishing theorem imply: 
If $Z$ is a twistor space over $n \mathbb{C P}^{2}$ with $n \leq 3$, then $Z$ has algebraic dimension three if and only if it has positive type.

The structure of these manifolds is, nowadays, fairly well-known (see [H2], [FK], [Po1], [KK] and [Po2]).

Since $c_{1}(Z)^{3}$ has the same sign as $4-n$, the case $n=4$ is somehow exceptional. According to results of F. Campana [C1] and C. LeBrun, Y.S.Poon [LeBP], in the case $n=4$ there exist twistor spaces $Z$ with $a(Z)=1$. In his paper [Po3] Poon expressed the algebraic dimension of a twistor space in terms of divisors whose restriction to twistor lines have degree one. In case $n=4$, the algebraic dimension is at least one. Poon's methods allow one to decide whether or not the algebraic dimension is three. See also [PP2].

Investigating the anti-canonical system on certain fundamental divisors (that is, elements in $\left|-\frac{1}{2} K\right|$ ), we shall be able to distinguish between algebraic dimension one and two. Our main technical result (Theorem 3.4) will be

Theorem. Let $Z$ be a twistor space of positive type over $4 \mathbb{C P}^{2}$ and $S$ a real fundamental divisor. Assume that $\left|-K_{S}\right|$ contains a smooth curve $C$. Let $N=N_{C \mid S}$ be the normal bundle of $C \subset S$. Then $N$ is of degree zero in $\operatorname{Pic}(C)$. We have:

$$
\begin{gathered}
a(Z) \leq 2 \text { and } \\
a(Z)=2 \Longleftrightarrow N \quad \text { is of finite order in the group } \operatorname{Pic}^{0}(C) .
\end{gathered}
$$

Using this result we obtain the main result of this paper (Theorem 4.1):

Theorem. There exist twistor spaces over $4 \mathbb{C P}^{2}$ having algebraic dimension two.

\section{Generalities}

This section collects known facts about twistor spaces. For proofs and more information the reader is referred to [ES], [H2], [Kr], [Po1].

Let $Z$ be a simply connected twistor space with $h^{2}\left(Z, \mathcal{O}_{Z}\right)=0$. Then $H^{i}(Z, \mathbb{Z})$ is a free $\mathbb{Z}$-module, which vanishes for $i$ odd. We define the integer $n$ by the equation $n+1=\operatorname{rank} H^{2}(Z, \mathbb{Z})$. The Chern numbers of $Z$ are:

$$
\begin{aligned}
c_{1}^{3} & =16(4-n), \\
c_{1} c_{2} & =24, \\
c_{3} & =2(n+2) .
\end{aligned}
$$

Cohomology of sheaves. For any twistor space we have $h^{3}\left(Z, \mathcal{O}_{Z}\right)=0$. By assumption, $h^{i}\left(Z, \mathcal{O}_{Z}\right)=0$ for $i=1,2$. In particular, we obtain an isomorphism of abelian groups, given by the first Chern class:

$$
\operatorname{Pic}(Z) \stackrel{\sim}{\longrightarrow} H^{2}(Z, \mathbb{Z})
$$

There exists a unique line bundle whose first Chern class is $\frac{1}{2} c_{1}$. We shall denote it by $K^{-\frac{1}{2}}$. Poon calls it the fundamental line bundle. The divisors in the linear system $\left|-\frac{1}{2} K\right|$ will be called fundamental divisors.

The degree of a line bundle $\mathcal{L} \in \operatorname{Pic}(Z)$ will be by definition the degree of its restriction to a real twistor line. For example, $\operatorname{deg}\left(K^{-\frac{1}{2}}\right)=2$. We obtain in this way a surjective degree map

$$
\operatorname{deg}: \operatorname{Pic}(Z) \rightarrow \mathbb{Z}
$$


From the above equations on Chern numbers we obtain, by applying the RiemannRoch theorem,

$$
\chi\left(Z, K^{-\frac{m}{2}}\right)=m+1+\frac{1}{3}(4-n) m(m+1)(m+2) .
$$

Vanishing theorems. The most important tool in classifying twistor spaces is Hitchin's vanishing theorem:

Theorem 2.1 (Hitchin [H1]). If $Z$ is of positive type, then we have for any $\mathcal{L} \in$ $\operatorname{Pic}(Z)$

$$
\operatorname{deg}(\mathcal{L}) \leq-2 \quad \Rightarrow \quad H^{1}(Z, \mathcal{L})=0 .
$$

On the other hand, since the twistor lines cover $Z$, we obtain:

$$
\operatorname{deg}(\mathcal{L}) \leq-1 \quad \Rightarrow \quad H^{0}(Z, \mathcal{L})=0 .
$$

By Serre-duality this gives the following important vanishing results:

$$
\begin{aligned}
& \operatorname{deg}(\mathcal{L}) \geq-2 \quad \Rightarrow \quad H^{2}(Z, \mathcal{L})=0 \\
& \operatorname{deg}(\mathcal{L}) \geq-3 \quad \Rightarrow \quad H^{3}(Z, \mathcal{L})=0
\end{aligned}
$$

\section{Computation of the algebraic Dimension}

Throughout this section we make the following assumptions: $Z$ is a twistor space over $4 \mathbb{C P}^{2}$, which is simply connected and of positive type. In particular: $h^{1}\left(Z, \mathcal{O}_{Z}\right)=h^{2}\left(Z, \mathcal{O}_{Z}\right)=0$.

From Section 2 we know

$$
h^{0}\left(K^{-\frac{m}{2}}\right)=m+1+h^{1}\left(K^{-\frac{m}{2}}\right) \quad \forall m \geq-1 ;
$$

in particular, $\operatorname{dim}\left|-\frac{1}{2} K\right| \geq 1$. The property $\left(-\frac{1}{2} K\right)^{3}=0$ will be crucial in what follows.

Lemma 3.1. The linear system $\left|-\frac{1}{2} K\right|$ contains a smooth real element.

Proof. This is proved in [PP1, Lemma 2.1] for the case that $\left|-\frac{1}{2} K\right|$ contains an irreducible element. If this is not the case, we would have infinitely many reducible elements in this linear system, hence infinitely many divisors of degree 1 in $Z$. But, by results of Kurke [Ku] and Poon [Po3], this occurs only in the case of LeBrun twistor spaces. For such twistor spaces the lemma is also true, which completes the proof.

Remark 3.2. The proof shows even more: If $Z$ is an arbitrary compact twistor space with $\operatorname{dim}\left|-\frac{1}{2} K\right| \geq 1$, then there exists a smooth real divisor in $\left|-\frac{1}{2} K\right|$.

Now let $S \in\left|-\frac{1}{2} K\right|$ be a real smooth divisor. Using the exact sequence

$$
0 \rightarrow K^{\frac{1}{2}} \rightarrow \mathcal{O}_{Z} \rightarrow \mathcal{O}_{S} \rightarrow 0
$$

and Hitchin's vanishing theorem we obtain $h^{i}\left(\mathcal{O}_{S}\right)=0$ for $i>0$. The adjunction formula gives $K_{S}^{-1}=K^{-\frac{1}{2}} \otimes \mathcal{O}_{S}$. From the exact sequence $0 \rightarrow \mathcal{O}_{Z} \rightarrow K^{-\frac{1}{2}} \rightarrow$ $K_{S}^{-1} \rightarrow 0$ we obtain

$$
H^{i}\left(Z, K^{-\frac{1}{2}}\right) \cong H^{i}\left(S, K_{S}^{-1}\right) \quad \forall i \geq 1
$$

and a surjective restriction map $H^{0}\left(Z, K^{-\frac{1}{2}}\right) \rightarrow H^{0}\left(S, K_{S}^{-1}\right)$ with one-dimensional kernel. In particular, $h^{0}\left(S, K_{S}^{2}\right)=h^{2}\left(S, K_{S}^{-1}\right)=h^{2}\left(Z, K^{-\frac{1}{2}}\right)=0$ and $h^{1}\left(S, \mathcal{O}_{S}\right)=$ 0 . Hence, by Castelnuovo's criterion, $S$ is a rational surface.

This leads to the following facts: 
(i) $\left|-\frac{1}{2} K\right|$ and $\left|-K_{S}\right|$ have the same base locus.

(ii) $h^{0}\left(K_{S}^{-1}\right)=h^{0}\left(K^{-\frac{1}{2}}\right)-1=1+h^{1}\left(K_{S}^{-1}\right) \geq 1$.

(iii) $\left(-K_{S}\right)^{2}=\left(-\frac{1}{2} K\right)^{3}=0$.

Lemma 3.3. If $\operatorname{dim}\left|-\frac{1}{2} K\right| \geq 2$, then $\left|-\frac{1}{2} K\right|$ has no basepoints $\Longleftrightarrow\left|-K_{S}\right|$ contains a smooth curve.

Proof. By assumption we have $\operatorname{dim}\left|-K_{S}\right| \geq 1$.

$(\Longrightarrow)$ is a consequence of Sard's or Bertini's theorem.

$(\Longleftarrow)$ Assume $\left|-\frac{1}{2} K\right|$ has basepoints. Since $\left(-K_{S}\right)^{2}=0$, the system $\left|-K_{S}\right|$ has no isolated basepoints. But since $\operatorname{dim}\left|-K_{S}\right| \geq 1$, there also exist moving components; hence $\left|-K_{S}\right|$ would contain reducible elements only. But all elements of $\left|-K_{S}\right|$ are connected since $h^{1}\left(K_{S}\right)=h^{1}\left(\mathcal{O}_{S}\right)=0$. This contradicts the existence of a smooth curve in $\left|-K_{S}\right|$.

Therefore, we have to distinguish three cases:

(i) $\left|-K_{S}\right|$ contains a smooth curve.

(ii) $\operatorname{dim}\left|-\frac{1}{2} K\right| \geq 2$ and it has basepoints.

(iii) $\operatorname{dim}\left|-\frac{1}{2} K\right|=1$ and $\left|-K_{S}\right|=\{C\}$ with a non-smooth curve $C$.

In the rest of this paper we shall discuss the first case.

Let $Z$ and $S$ be as above and $C \in\left|-K_{S}\right|$ a smooth curve. By adjunction we obtain $K_{C}=\mathcal{O}_{C}$; hence $C$ is an elliptic curve. Let $N=N_{C \mid S}=K_{S}^{-1} \otimes \mathcal{O}_{C}$ denote the normal bundle of $C$ in $S$. From $\left(-K_{S}\right)^{2}=0$ we obtain: $\operatorname{deg} N=0$.

Having this in mind, we formulate the following

Theorem 3.4. Let $Z$ be a twistor space of positive type over $4 \mathbb{C P}^{2}$ and $S$ a real and smooth fundamental divisor. Assume that $\left|-K_{S}\right|$ contains a smooth curve $C$. Denote the order of $N=K_{S}^{-1} \otimes \mathcal{O}_{C}$ in the group $\operatorname{Pic}(C)$ by $\tau$. This number is related to the algebraic dimension $a(Z)$ in the following way:

$$
\begin{aligned}
\tau=\infty & \Longleftrightarrow a(Z)=1, \\
\tau<\infty & \Longleftrightarrow a(Z)=2 .
\end{aligned}
$$

If $1 \leq m<\tau$, we have $h^{0}\left(K^{-\frac{m}{2}}\right)=m+1$ and $h^{1}\left(K^{-\frac{m}{2}}\right)=0$.

If $1 \leq m<\tau$ and $i \geq 0$, we have $h^{i}\left(K_{S}^{-m}\right)=h^{i}\left(\mathcal{O}_{S}\right)$.

If $\tau<\infty$, then we have, furthermore:

$\left|-\frac{\tau}{2} K\right|$ has no basepoints, $h^{0}\left(K^{-\frac{\tau}{2}}\right)=\tau+2 \quad$ and $\quad h^{1}\left(K^{-\frac{\tau}{2}}\right)=h^{1}\left(K_{S}^{-\tau}\right)=1$.

Proof. We shall basically rely on the result of Poon [Po2] that $a(Z)=\kappa\left(Z, K^{-1}\right)$ (the Iitaka dimension of the anti-canonical line bundle). Therefore, from $h^{0}\left(K^{-\frac{1}{2}}\right)$ $\geq 2$ we immediately see: $a(Z) \geq 1$.

Consider the following two exact sequences: $(m \in \mathbb{Z})$

$$
\begin{gathered}
0 \rightarrow K_{S}^{-(m-1)} \rightarrow K_{S}^{-m} \rightarrow N^{\otimes m} \rightarrow 0, \\
0 \rightarrow K^{-\frac{m-1}{2}} \rightarrow K^{-\frac{m}{2}} \rightarrow K_{S}^{-m} \rightarrow 0 .
\end{gathered}
$$

Observe that $h^{0}\left(N^{\otimes m}\right)=h^{1}\left(N^{\otimes m}\right)=1$ if $N^{\otimes m} \cong \mathcal{O}_{C}$. In all other cases these numbers vanish. This is because $C$ is elliptic and $\operatorname{deg} N=0$.

If $1 \leq m<\tau$, then $h^{0}\left(N^{\otimes m}\right)=h^{1}\left(N^{\otimes m}\right)=0$; hence (7) gives: $h^{i}\left(K_{S}^{-(m-1)}\right)=$ $h^{i}\left(K_{S}^{-m}\right)$ and so by induction

$$
h^{i}\left(K_{S}^{-m}\right)=h^{i}\left(\mathcal{O}_{S}\right) \quad \forall 1 \leq m<\tau .
$$


Using $h^{1}\left(\mathcal{O}_{Z}\right)=0$, the sequence (8) yields, by induction on $m \geq 1, h^{0}\left(K^{-\frac{m}{2}}\right)=$ $1+h^{0}\left(K^{-\frac{m-1}{2}}\right)$ and $h^{1}\left(K^{-\frac{m}{2}}\right)=0$ and so

$$
h^{0}\left(K^{-\frac{m}{2}}\right)=m+1 \quad \forall 1 \leq m<\tau .
$$

In particular, if $\tau=\infty$, we obtain $a(Z)=\kappa\left(Z, K^{-1}\right)=1$.

Assume for the rest of the proof $\tau<\infty$.

With the above computations the cohomology sequences of (7) and (8) with $m=\tau$ yield $h^{0}\left(K_{S}^{-\tau}\right)=2, h^{1}\left(K_{S}^{-\tau}\right)=1$ and

$$
\begin{gathered}
h^{0}\left(K^{-\frac{\tau}{2}}\right)=h^{0}\left(K^{-\frac{\tau-1}{2}}\right)+h^{0}\left(K_{S}^{-\tau}\right)=\tau+2, \\
h^{1}\left(K^{-\frac{\tau}{2}}\right)=h^{1}\left(K_{S}^{-\tau}\right)=1 .
\end{gathered}
$$

Next we prove that $\left|-\frac{\tau}{2} K\right|$ has no basepoints. Since $\tau S \in\left|-\frac{\tau}{2} K\right|$, we see from the exact sequence

$$
0 \rightarrow H^{0}\left(K^{-\frac{\tau-1}{2}}\right) \rightarrow H^{0}\left(K^{-\frac{\tau}{2}}\right) \rightarrow H^{0}\left(K_{S}^{-\tau}\right) \rightarrow 0
$$

that $\left|-\frac{\tau}{2} K\right|$ and $\left|-\tau K_{S}\right|$ have the same base locus.

But the exact sequence

$$
0 \rightarrow H^{0}\left(K_{S}^{-(\tau-1)}\right) \rightarrow H^{0}\left(K_{S}^{-\tau}\right) \rightarrow H^{0}\left(\mathcal{O}_{C}\right) \rightarrow 0
$$

tells us that there is a section in $H^{0}\left(K_{S}^{-\tau}\right)$ which does not vanish at any point of $C$. Hence, the base locus of $\left|-\tau K_{S}\right|$ is empty.

It remains to show $a(Z) \geq 2$.

Let $\Phi_{\tau}: Z \rightarrow \mathbb{P}^{\tau+1}$ be the morphism defined by the linear system $\left|-\frac{\tau}{2} K\right|$. Let $X_{\tau} \subset \mathbb{P}^{\tau+1}$ be the image of $\Phi_{\tau}$. Assume $a(Z)=1$; then by $a(Z)=\kappa\left(Z, K^{-\frac{1}{2}}\right)$ we have $\operatorname{dim} X_{\tau}=1$. By definition of $\Phi_{\tau}$ the curve $X_{\tau}$ is not contained in a proper linear subspace of $\mathbb{P}^{\tau+1}$. Using a well-known result $([\mathrm{H}, \S 18])$, this implies $\operatorname{deg} X_{\tau} \geq \tau+1$. Since $X_{\tau}$ is irreducible and reduced, a generic hyperplane section meets this curve at exactly $\operatorname{deg} X_{\tau}$ distinct points. Hence, the generic element in $\left|-\frac{\tau}{2} K\right|$ is the sum of $\operatorname{deg} X_{\tau}$ disjoint, but algebraically (even linearly, since $\pi_{1}(Z)=$ 0 forces $X_{\tau}$ to be a rational curve) equivalent divisors in $Z$. Thus, alternatively, because $\operatorname{Pic}(Z) \cong H^{2}(Z, \mathbb{Z})$ is a free abelian group, we obtain $-\frac{\tau}{2} K=\delta K_{0}$. Here $K_{0}$ is a fibre of $\Phi_{\tau}$ and $\delta=\operatorname{deg} X_{\tau} \geq \tau+1$. Computation of the degree gives $2 \tau=\delta \operatorname{deg} K_{0}$. Therefore, $\delta=2 \tau$ and $\operatorname{deg} K_{0}=1$. Then there were infinitely many divisors of degree 1 in $Z$, leading again to the case of LeBrun twistor spaces. But for such twistor spaces we have $h^{0}\left(K^{-\frac{1}{2}}\right)=4$, in contradiction to our computation. This contradiction shows: $\operatorname{dim} X_{\tau} \geq 2$; hence $a(Z) \geq 2$.

To finish the proof, we should show $a(Z) \leq 2$. We can easily derive this from the sequences (7) and (8) as follows: From (8) we obtain, for all $m \in \mathbb{Z}$,

$$
P(m):=h^{0}\left(K^{-\frac{m}{2}}\right)-h^{0}\left(K^{-\frac{m-1}{2}}\right) \leq h^{0}\left(K_{S}^{-m}\right)
$$

and from (7)

$$
h^{0}\left(K_{S}^{-m}\right)-h^{0}\left(K_{S}^{-(m-1)}\right) \leq h^{0}\left(N^{\otimes m}\right) \leq 1 .
$$

Thus $P(m)$ grows at most linearly in $m$. Hence, $h^{0}\left(K^{-\frac{m}{2}}\right)$ grows at most quadratically. This means (cf. [C1]): $a(Z)=\kappa\left(Z, K^{-\frac{1}{2}}\right) \leq 1+\kappa\left(S, K_{S}^{-1}\right) \leq 2+\kappa(C, N) \leq 2$, as desired. 


\section{THE EXISTENCE THEOREM}

In this section we apply the results of Section 3 to prove the following theorem:

Theorem 4.1. There exist twistor spaces over $4 \mathbb{C P}^{2}$ having algebraic dimension two.

One method to establish the existence of twistor spaces is to study the deformation theory of known twistor spaces. It was developed by Donaldson-Friedman [DonF] (see also Campana [C1] and LeBrun [LeB2]). We use the following theorem:

Theorem 4.2 ([C1], [C3], [DonF], [PP2]). Let Z be a LeBrun twistor space, $n \geq$ 4 and $S \in\left|-\frac{1}{2} K\right|$ a smooth real divisor. Then: Any real member of a small deformation of $Z$ is again a twistor space. Furthermore, any small deformation of $S$ with real structure is induced by a deformation of $Z$ in the sense that the deformed surfaces are members of the fundamental system of the deformed twistor spaces.

We prove Theorem 4.1 by constructing a small deformation with real structure of a smooth rational surface $S=S_{0} \in\left|-\frac{1}{2} K\right|$ in a certain twistor space. We shall prove that there exist real deformed surfaces containing in their anticanonical system a smooth curve whose normal bundle is a torsion element in the Picard group. The result then follows by Theorems 3.4 and 4.2 .

We shall use the explicitly known twistor spaces, which are discovered by C. LeBrun [LeB1] and investigated by H. Kurke [Ku]. They are described as modifications of conic-bundles over $\mathbb{P}^{1} \times \mathbb{P}^{1}$. Let us recall the structure of general real surfaces $S \in\left|-\frac{1}{2} K\right|$ in generic LeBrun twistor spaces [Ku], [LeB1]: $S$ contains a twistor line $F \subset S$. On $S$ we have $\left(F^{2}\right)=0$ and $\operatorname{dim}|F|=1$. Since $\left(K_{S}\right)^{2}=0$, we obtain: the morphism $\pi: S \rightarrow \mathbb{P}^{1}$ defined by $|F|$ exhibits $S$ as a successive blowup of eight points on a ruled surface. More precisely, we know (cf. [PP2]) that $\pi$ factors over a blow-up $S \rightarrow \mathbb{P}^{1} \times \mathbb{P}^{1}$, which is compatible with real structures. The real structure on $\mathbb{P}^{1} \times \mathbb{P}^{1}$ is given by the antipodal map on the first factor and the usual real structure on the second factor. The blown-up set is real and consists of eight distinct points, lying on two conjugate fibres of the first projection. The fibres of the second projection correspond to the elements of $|F|$. This situation is illustrated by the following picture:

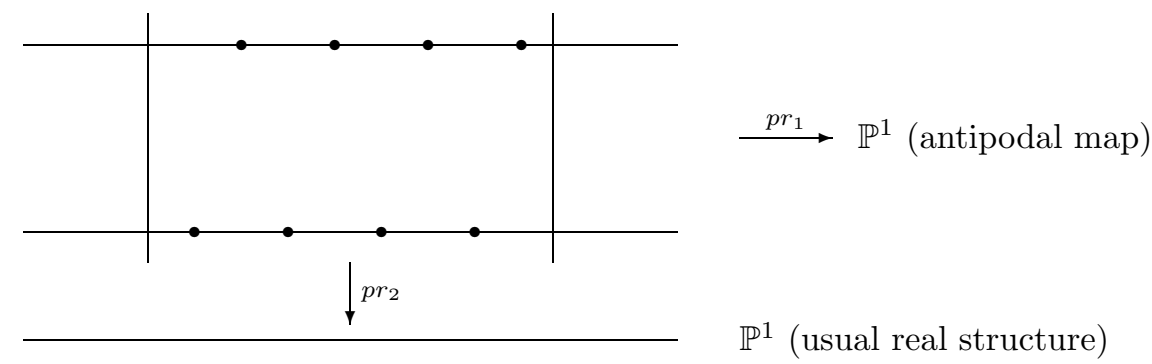

None of the blown-up points lie on a real fibre of the second projection since all real elements in $|F|$ are irreducible (real twistor lines).

To begin our construction, we fix a blowing-up $S=S_{0} \rightarrow \mathbb{P}^{1} \times \mathbb{P}^{1}$ of eight points $\left\{P_{1}, \ldots, P_{8}\right\}$ as described above and choose a curve $C_{0} \subset \mathbb{P}^{1} \times \mathbb{P}^{1}$ of type $(2,2)$ containing $\left\{P_{1}, \ldots, P_{8}\right\}$ as smooth points.

Consider the universal family of curves of type $(2,2)$ on $\mathbb{P}^{1} \times \mathbb{P}^{1}$, which is given as $\mathcal{C}=\{(C, x) \mid x \in C\} \subset|\mathcal{O}(2,2)| \times\left(\mathbb{P}^{1} \times \mathbb{P}^{1}\right)$. The family $\mathcal{C} \rightarrow B:=|\mathcal{O}(2,2)|$ is a 
deformation of $C_{0}$ with basis $B \cong \mathbb{P}^{8}$. By $0 \in B$ we denote the point corresponding to $C_{0}$ and by $C_{t} \subset \mathbb{P}^{1} \times \mathbb{P}^{1}$ the fibre over $t \in B$.

By $B^{\prime} \rightarrow B$ we denote the eight-fold fibre product $B^{\prime}=\mathcal{C} \times{ }_{B} \mathcal{C} \times{ }_{B} \cdots \times{ }_{B} \mathcal{C} \rightarrow$ $B$. Then the pulled back family $\mathcal{C}^{\prime}:=\mathcal{C} \times_{B} B^{\prime} \rightarrow B^{\prime}$ has eight natural sections $P_{1}, \ldots, P_{8}$, given by the projections $p r_{i}: B^{\prime} \rightarrow \mathcal{C}$.

We denote by $\bar{B}$ the quotient under the natural action of the symmetric group $S_{8}$ on the open set of eight-tuples of pairwise distinct points (which is the complement of all twofold diagonals). The family $\mathcal{C}^{\prime} \rightarrow B^{\prime}$ descends to a family $\overline{\mathcal{C}} \rightarrow \bar{B}$. Since the action of $S_{8}$ is fibre preserving for $B^{\prime} \rightarrow B$, we obtain a morphism $\bar{B} \rightarrow B$. The union of the eight sections $P_{1}, \ldots, P_{8}$ is $S_{8}$-invariant; hence over $\bar{B}$ we obtain a family of subschemes of $\mathbb{P}^{1} \times \mathbb{P}^{1}$ of length eight $Z=\bigcup_{i=1}^{8} P_{i}(\bar{B}) \subset \overline{\mathcal{C}} \subset \bar{B} \times\left(\mathbb{P}^{1} \times \mathbb{P}^{1}\right) \rightarrow$ $\bar{B}$. The given configuration of eight points on $C_{0}$ defines a point $0 \in \bar{B}$. If we blow up $\bar{B} \times\left(\mathbb{P}^{1} \times \mathbb{P}^{1}\right)$ along $Z$, we obtain a family of surfaces $\mathcal{S} \rightarrow \bar{B}$ with fibre $S_{0}=S$ over $0 \in \bar{B}$.

The given real structure on $\mathbb{P}^{1} \times \mathbb{P}^{1}$ defines a real structure on $B$ and $\mathcal{C}$, such that $\mathcal{C} \rightarrow B$ is equivariant. Therefore, we finally obtain a real structure on $\bar{B}, \overline{\mathcal{C}}, Z$ and $\mathcal{S} \rightarrow \bar{B}$, such that all maps considered before are equivariant.

Hence, by Theorem 4.2 , there exists a neighbourhood $\bar{B}^{*}$ of $0 \in \bar{B}$ in the analytic topology, such that any real $S_{t}$ sits in a twistor space as a fundamental divisor. We can choose $\bar{B}^{*}$ in such a way that for all $t \in \bar{B}^{*}$ the set of eight different points $Z_{t}$ consists of smooth points of $C_{t}$. Therefore, the strict transform of $C_{t}$ in $S_{t}$ will be isomorphic to $C_{t}$ and we shall denote it also by $C_{t}$.

We wish now to study the order of the normal bundle $N_{t}=N_{C_{t} \mid S_{t}}$ of $C_{t} \subset S_{t}$.

Let us denote by $\bar{U} \subset \bar{B}^{*}$ the open subset of those points $t \in \bar{B}^{*}$ with smooth $C_{t}$. Its image $U \subset B$ is also open since projections and flat maps are open. Since $N_{t} \cong \mathcal{O}(2,2) \otimes \mathcal{O}_{C_{t}}\left(-Z_{t}\right) \in \operatorname{Pic}^{0}\left(C_{t}\right)$, we obtain a morphism $\eta: \bar{U} \rightarrow \operatorname{Pic}^{0}(\mathcal{C} \mid U)$ with $\eta(t)=N_{t}$. This morphism is compatible with real structures.

To achieve our theorem, we have to show the existence of a real point in $\bar{U}(\mathbb{R})$, which is sent by $\eta$ to a point of finite order. This will follow from

Lemma 4.3. Let $E$ be an elliptic curve (over $\mathbb{C}$ ) with a real structure and $\mathcal{L}$ a line bundle on $E$ which has degree 8 and is real. Let $\Delta \subset E \times \cdots \times E / S_{8}$ be the moduli space of sets of eight distinct (closed) points on $E$. Let $\eta: \Delta \rightarrow \operatorname{Pic}^{0}(E)$ be the map defined by $\eta\left(\left\{x_{1}, \ldots, x_{8}\right\}\right)=\mathcal{L} \otimes \mathcal{O}_{E}\left(-\sum_{i=1}^{8} x_{i}\right)$. Then we have:

(i) The real structure on $E$ induces one on $\Delta$ and $\operatorname{Pic}^{0}(E)$, such that $\eta$ is equivariant.

(ii) The real points $\operatorname{Pic}^{0}(E)(\mathbb{R})$ form a real one-dimensional Lie group. The points of finite order form a dense subset in this group.

(iii) $\eta: \Delta \rightarrow \operatorname{Pic}^{0}(E)$ and $\eta_{\mathbb{R}}: \Delta(\mathbb{R}) \rightarrow \operatorname{Pic}^{0}(E)(\mathbb{R})$ are submersions and are, therefore, open maps.

Proof. Observe first that the precise definition of $\eta$ as a holomorphic map comes from the universality property of the variety $\operatorname{Pic}^{0}(E)$.

(i) The real structure on $\operatorname{Pic}^{0}(E)$ is defined by $\mathcal{M} \mapsto \sigma^{*} \overline{\mathcal{M}}$. (We denote by $\overline{\mathcal{M}}$ the complex conjugate line bundle and $\sigma$ is the antiholomorphic involution on $E$ defining the real structure.) Since $\mathcal{L}$ is, by assumption, a fixed point of this real structure, the map $\eta$ is, by definition, equivariant. 
(ii) Since $\mathcal{O}_{E} \in \operatorname{Pic}^{0}(E)$ is a real point, the subset $\operatorname{Pic}^{0}(E)(\mathbb{R})$ of real points forms a real one-dimensional submanifold of $\operatorname{Pic}^{0}(E)$. Since the formation of tensor products and duals is compatible with the real structure, $\operatorname{Pic}^{0}(E)(\mathbb{R})$ forms a subgroup of $\operatorname{Pic}^{0}(E)$. Since $U(1)$ is the unique connected compact one-dimensional real abelian Lie group, the connected component of $\mathcal{O}_{E}$ in $\operatorname{Pic}^{0}(E)(\mathbb{R})$ must be isomorphic to $U(1)$. But the set of points of finite order in this group is dense. By compactness, $\operatorname{Pic}^{0}(E)(\mathbb{R})$ has only a finite number of connected components. Then it is easy to see that the set of points of finite order is dense in $\operatorname{Pic}^{0}(E)(\mathbb{R})$.

(iii) Let $\widetilde{\Delta} \subset E \times \cdots \times E$ be the preimage of $\Delta$, that is, the set of eight-tuples of pairwise distinct points. Since $E$ is elliptic, by Riemann-Roch there exist eight points $Q_{1}, \ldots, Q_{8} \in E$ with $\mathcal{L} \cong \mathcal{O}_{E}\left(\sum_{i=1}^{8} Q_{i}\right)$. If we define $\eta_{i}: E \rightarrow \operatorname{Pic}^{0}(E)$ by $\eta_{i}(P):=\mathcal{O}_{E}\left(Q_{i}-P\right)$ and $\widetilde{\eta}=\left(\eta_{1}, \ldots, \eta_{8}\right)$, we obtain a commutative diagram:

$$
\begin{array}{cccc}
\widetilde{\Delta} & \stackrel{\tilde{\eta}}{\longrightarrow} & \operatorname{Pic}^{0}(E) \times \cdots \times \operatorname{Pic}^{0}(E) \\
\downarrow & & \downarrow \text { mult } \\
\Delta & \stackrel{\eta}{\longrightarrow} & \operatorname{Pic}^{0}(E) .
\end{array}
$$

Since all $\eta_{i}$ are isomorphisms, $\widetilde{\eta}$ is an open embedding, hence a submersion. Since multiplication is always a submersion, we have the same property for $\eta$.

Finally, since $\eta$ is equivariant, its tangent map has the same property. But, if a complex linear map is compatible with real structures and surjective, the corresponding map on real subspaces is also surjective, since it can be described by the same matrix. So we obtain that $\eta_{\mathbb{R}}$ is a submersion. Submersions are open by the implicit function theorem.

Proof of Theorem 4.1. We take any $t \in U(\mathbb{R})$ and apply Lemma 4.3 to $E=C_{t}$ and $\mathcal{L}=\mathcal{O}(2,2) \otimes \mathcal{O}_{C_{t}}$. This gives the existence of a point $\bar{t} \in \bar{U}(\mathbb{R})$ over $t$, defining a torsion point $\eta(\bar{t})$. The result follows now from Theorem 3.4.

Open Problem. Which values for $\tau$ can be realized by twistor spaces over $4 \mathbb{C P}^{2}$ ?

Our proof of Theorem 4.1 only shows that large values of $\tau$ really occur.

\section{ACKNOWLEDGEMENT}

The second author thanks the Université de Nancy for its hospitality during his stay in the autumn of 1994 . This stay was supported by INTERREG.

\section{REFERENCES}

[AHS] M.F. Atiyah, N.J. Hitchin, I.M. Singer: Self-duality in four-dimensional Riemannian geometry, Proc. Roy. Soc. London Ser. A 362 (1978) 425-461. MR 80d:53023

[C1] F. Campana: The class $\mathcal{C}$ is not stable by small deformations, Math. Ann. 290 (1991) 19-30. MR 93d:32047

[C2] F. Campana: On Twistor Spaces of the Class C, J. Diff. Geom. 33 (1991) 541-549. MR 92g:32059

[C3] F. Campana: The class $\mathcal{C}$ is not stable by small deformations II, Contemp. Math. 162 (1994) 65-76. MR 95i:32026

[Don] S. Donaldson: An application of gauge theory to the topology of 4-manifolds, J. Diff. Geom. 18 (1983) 279-315. MR 85c:57015

[DonF] S. Donaldson, R. Friedman: Connected sums of self-dual manifolds and deformations of singular spaces, Nonlinearity 2 (1989) 197-239. MR 90e:32027 
[ES] M.G. Eastwood, M.A. Singer: The Fröhlicher Spectral Sequence on a Twistor Space, J. Diff. Geom. 38 (1993) 653-669. MR 94k:32050

[F] M. Freedman: The topology of four-dimensional manifolds, J. Diff. Geom. 17 (1982) 357-454. MR 84b:57006

[FK] T. Friedrich, H. Kurke: Compact four-dimensional self-dual Einstein manifolds with positive scalar curvature, Math. Nach. 106 (1982) 271-299. MR 84b:53043

[G] P. Gauduchon: Structures de Weyl et théorèmes d'annulation sur une variété conforme autoduale, Ann. Scuola Norm. Sup. Pisa 18 (1991) 563-629. MR 93d:32046

$[\mathrm{H}] \quad$ J. Harris: Algebraic Geometry, Graduate Texts in Mathematics 133, Springer-Verlag, 1992. MR 93j:14001

[H1] N.J. Hitchin: Linear field equations on self-dual spaces, Proc. Roy. Soc. London Ser. A 370 (1980) 173-191. MR 81i:81057

[H2] N.J. Hitchin: Kählerian twistor spaces, Proc. Lond. Math. Soc., III Ser. 43 (1981) 133-150. MR 84b:32014

[Kr] B. Kreußler: Small resolutions of double solids, branched over a 13-nodal quartic surface, Annals of Global Analysis and Geometry 7 (1989) 227-267. MR 91b:14011

[KK] B. Kreußler, H. Kurke: Twistor spaces over the connected sum of 3 projective planes, Compositio Math. 82 (1992) 25-55. MR 93d:32049

$[\mathrm{Ku}]$ H. Kurke: Classification of twistor spaces with a pencil of surfaces of degree 1, Part I, Math. Nachr. 158 (1992) 67-85. MR 94k:32051

[LeB1] C. LeBrun: Explicit self-dual metrics on $\mathbb{C P}^{2} \# \ldots \# \mathbb{C P}^{2}$, J. Diff. Geom. 34 (1991) 223-253. MR 92g:53040

[LeB2] C. LeBrun: Twistors, Kähler manifolds, and bimeromorphic geometry. I, J. AMS 5 (1992) 289-316. MR 92m:32052

[LeBP] C. LeBrun, Y.S. Poon: Twistors, Kähler manifolds, and bimeromorphic geometry. II, J. AMS 5 (1992) 317-325. MR 92m:32053

[PP1] H. Pedersen, Y.S. Poon: Self-duality and differentiable structures on the connected sum of complex projective planes, Proc. Amer. Math. Soc. 121 (1994) 859-864. MR 94i:32049

[PP2] H. Pedersen, Y.S. Poon: A relative deformation of Moishezon twistor spaces, J. Alg. Geom. 3 (1994) 685-701. MR 95j:32045

[PP3] H. Pedersen, Y.S. Poon: Equivariant connected sums of compact self-dual manifolds, Math. Ann. 301 (1995) 717-749. MR 95m:53069

$[\mathrm{Pe}] \quad$ R. Penrose: Nonlinear gravitons and curved twistor theory, General Relativity and Gravitation 7 (1976) 31-52. MR 55:11905

[Pon1] M. Pontecorvo: Hermitian Surfaces and a Twistor Space of Algebraic Dimension 2, Proc. Amer. Math. Soc. 113 (1991) 177-186. MR 91k:32028

[Pon2] M. Pontecorvo: Algebraic dimension of twistor spaces and scalar curvature of anti-selfdual metrics, Math. Ann. 291 (1991) 113-122. MR 92j:32113

[Po1] Y.S. Poon: Compact self-dual manifolds with positive scalar curvature, J. Diff. Geom. 24 (1986) 97-132. MR 88b:32022

[Po2] Y.S. Poon: Algebraic dimension of twistor spaces, Math. Ann. 282 (1988) 621-627. MR 90f: 32029

[Po3] Y.S. Poon: On the algebraic structure of twistor spaces, J. Diff. Geom. 36 (1992) 451-491. MR 94a:32045

[Sch] R. Schoen: Conformal deformation of a Riemannian metric to constant scalar curvature, J. Diff. Geom. 20 (1984) 479-495. MR 86i:58137

[V] M. Ville: Twistor examples of algebraic dimension zero treefolds, Invent. math. 103 (1991) 537-545. MR 92c:32034

Department of Mathematics, Université de Nancy, F-54 506 Vandeuvre les Nancy, FRANCE

E-mail address: Frederic.Campana@iecn.u-nancy.fr

Department of Mathematics, Universität Kaiserslautern, D-67653 Kaiserslautern, GERMANY

E-mail address: kreusler@mathematik.uni-kl.de 